\title{
Vaginal preparation with povidone iodine solution before caesarean section and its impact on the post-operative infectious morbidity
}

\author{
Savita Chandra, Vandana Singh*
}

Department of Obstetrics and Gynecology, Era’s Lucknow Medical College and Hospital, Lucknow, Uttar Pradesh, India

Received: 17 May 2021

Accepted: 15 June 2021

\author{
*Correspondence: \\ Dr. Vandana Singh, \\ E-mail: vandanasingh.red@gmail.com
}

Copyright: (c) the author(s), publisher and licensee Medip Academy. This is an open-access article distributed under the terms of the Creative Commons Attribution Non-Commercial License, which permits unrestricted non-commercial use, distribution, and reproduction in any medium, provided the original work is properly cited.

\begin{abstract}
Background: A prospective randomized control trial (RCT) was conducted in the department of obstetrics and gynecology. The aim of the study was to evaluate the effect of pre-operative vaginal preparation with $1 \%$ povidone iodine solution on the post-operative caesarean infectious morbidity.

Methods: 72 women were enrolled in the experimental group who received $1 \%$ povidone iodine vaginal wash and 72 in the control group who did not receive any vaginal wash. The investigators were blinded to the computer-generated random number table and to the allocation group. The clinical data was recorded and after its completion, the allocation group was revealed to the investigator. The data was tabulated and statistically analyzed using SPSS 21.

Results: Age, parity, BMI, gestational age, blood loss, and operative time between the two groups was comparable. Pre-operative povidone iodine vaginal wash was more effective when membranes were intact versus ruptured. With intact membranes, statistical significance was reached for postoperative fever, seroma, endometritis, gaped incisional site, and foul discharge with $\mathrm{p}$ value $0.012,0.012,0.013,0.027$ and 0.012 respectively, between group A and $\mathrm{B}$. The overall infectious morbidity in the 144 subjects showed statistical significance for endometritis, and foul-smelling lochia with $\mathrm{p}$ value 0.049 and 0.024 respectively; urinary tract infection, hospital stay duration, and incisional wound dehiscence were higher in group B versus group A, the difference in these parameters did not reach statistical significance.

Conclusions: Pre-operative vaginal cleansing with $1 \%$ povidone iodine reduced post-operative infectious morbidity more effectively in those with intact membranes than ruptured membranes.
\end{abstract}

Keywords: Post caesarean infectious morbidity, Pre-operative vaginal wash, Povidone iodine

\section{INTRODUCTION}

Over the passing years caesarean section has become relatively safe but despite this, postoperative infectious morbidity continues to be a matter of concern. Further, over the last two decades, the dramatic increase in the cesarean section rates worldwide has made it a major surgical procedure of high volume. Additionally, caesarean section is also the single most important risk factor for maternal infectious morbidity.

The post-operative caesarean infectious morbidity predominantly involves febrile morbidity, surgical site infections, infection of the uterus and urinary tract, and less commonly other systems. Surgical site infection affects 3 to $15 \%$ of caesarean deliveries worldwide. ${ }^{1-3}$ One in ten women develop incision site infection and $25 \%$ develop endometritis. ${ }^{4}$ These risks are even higher in low resource settings lacking comprehensive obstetric care. ${ }^{5}$

Physiologically, the vagina has several microorganisms and during vaginal or caesarean delivery the delicate vaginal microbiota balance gets disturbed and if disturbed beyond a certain threshold, yet unknown, the vagina becomes a potential source of ascending infection. Antiseptic cleaning of the abdomen, use of autoclaved 
drapes and instruments, surgical gowns and gloves and use of pre-incision/intra-operative prophylactic antibiotics are the modalities to reduce postoperative infectious morbidity. Not-withstanding these measures, postoperative infectious morbidity remains a challenge.

The Centre for Disease Control (CDC) and WHO, endorsed the importance of preoperative vaginal preparation and use of prophylactic antibiotics in their recent guidelines. ${ }^{6,7}$ With this in mind the study was planned.

\section{METHODS}

A prospective randomized control trial (RCT) was conducted in the department of obstetrics and gynecology, Era's Lucknow Medical College and Hospital, India, from November 2018 to February 2020. Approval for the study was obtained from the Institution's Ethics Committee. Informed consent was taken from each subject before enrolment into the study. The calculated sample size was 144 i.e.; 72 each in the experimental group (group A) and the control group (group B)

Women planned for elective caesarean section as well as those for emergency caesarean section were included in the study. Those listed for caesarean section but with any of the following conditions were excluded i.e. ruptured membranes for $>6 \mathrm{hrs}$, chorioamnionitis, fever, history of allergy to iodine or other medications, gestational diabetes mellitus, obesity (BMI>30), severe anemia, urinary and respiratory tract infection.

Randomization was accomplished by computer-generated random number table to allocate the 2 groups. Group A was the experimental group 'vaginal wash with povidone iodine' and group B was the control group with 'no wash'.

144 index cards were prepared with one of the two instructions written on the index card i.e.; either 'vaginal wash with povidone iodine' or 'no wash'. Each card was placed in an individual opaque envelope. The envelopes were then sealed, and sequentially numbered from serial number 1 to 144 .

Prior to the caesarean section only one envelope was removed in serial order and opened by one member of the surgical team to note the instruction. The admission registration number of that specific patient was then written on the surface of the envelope and the index card placed into the envelope and firmly sealed.

Just prior to the preoperative urinary catheterization vaginal douching was done with $1 \%$ povidone iodine solution/or not done as per the noted instruction. The study investigators were blinded to the computer-generated random number table and to the allocation group to eliminate selection bias. Uniformity was maintained with regard to the abdominal wall surgical site skin preparation using standard chlorhexidine and betadine solution for all
144 subjects of the study as well as for the pre-incision antibiotic prophylaxis protocol prior to caesarean section for all the 144 subjects enrolled into the study.

Post-operatively, the following day, the study investigator noted the admission registration number written on the surface of the closed envelope, and through the registration number identified the patient. The admission registration number was recorded on the study proforma. The patient was followed post operatively, and the clinical details including the postoperative infectious morbidity of each patient was recorded on the proforma.

After recording the clinical data of all the 144 subjects, the sealed envelopes were opened in the presence of two senior faculty, unconnected with the study, and the 144 proformas then placed into their respective group i.e.; group A, (experimental) or group B (control) The clinical data was then tabulated and analyzed. Statistical analysis was done using SPSS version 21.

\section{RESULTS}

In this study, a total of 144 women were enrolled. The Experimental and the Control group each had 72 subjects. There was no significant difference between the two groups regarding mean age, mean BMI, mean gestational age, mean duration of rupture of membranes, number of pelvic examinations before surgery, mean amount of blood loss, mean preoperative and postoperative hemoglobin. All the parameters were comparable with no statistical significance (Table 1).

Among the women who had intact membranes at the time of caesarean section, postoperative incidence of fever in the control group B was $16.7 \%$ versus $2.0 \%$ in the experimental group, statistically significant with $p$ value $=0.012$ (Table 2). Endometritis was not seen in group A while it occurred in $11.7 \%$ in group B, statistically significant $\mathrm{p}$ value $=0.013$; seroma was seen in $2 \%$ in group $\mathrm{A}$ and $16.7 \%$ in group $\mathrm{B}, \mathrm{p}$ value $<0.012$ which is statistically significant. Similarly, gaped stitch line, and foul-smelling lochia were also more in the control group B as compared to the experimental group $\mathrm{A}$ and the difference reached statistical significance, with $\mathrm{p}$ value 0.027 and 0.012 respectively.

As from Table 2, urinary tract infection, hospital readmission, was more in group B as compared to group A, but the difference was not statistically significant. Thus, for women with intact membranes prior to caesarean section statistical significance was reached for febrile morbidity, endometritis, seroma, gaped surgical incisional site, and foul-smelling vaginal discharge. Coming to the infectious morbidity in women who had rupture of membranes at the time of caesarean section. In our study there were 23 women in group A and 12 women in group $B$ who had ruptured membranes at the time of caesarean section (Table 3). Interestingly, the incidence of the various infectious morbidity parameters observed were 
higher in the experimental group as compared to the control group but the difference did not reach statistical significance for any parameter. This suggests that ruptured membranes were an important variable for postoperative infectious morbidity. Further, it can also be deduced that the preoperative vaginal preparation with povidone iodine was not so effective in reducing the postoperative infectious morbidity in subjects with ruptured membranes prior to caesarean section compared to those with intact membranes. When the overall caesarean infectious morbidity was tabulated for both ruptured and intact membranes, the incidence of fever, endometritis, seroma, gaped incisional wound, urinary tract infection, foul smelling lochia, and hospital stay were lower in the experimental group as compared to the control, but the difference in these parameters did not reach statistical significance. However, statistical significance was reached for endometritis and foul-smelling lochia with $p$ value $<0.049$ and 0.024 respectively (Table 4).

Table 1: Comparison of the clinical profile of group A and B.

\begin{tabular}{|llll|}
\hline Variables & Group A $(\mathbf{N}=\mathbf{7 2})$ & Group B $(\mathrm{N}=72)$ & Statistical significance \\
\hline Mean age \pm SD $($ years $)$ & $27.50 \pm 5.26(18-38)$ & $27.50 \pm 5.24(19-45)$ & $\mathrm{t}=0, \mathrm{p}=1$ \\
\hline Mean BMI \pm SD $\left(\mathbf{k g} / \mathbf{m}^{\mathbf{2}}\right)$ & $23.41 \pm 2.52$ & $23.87 \pm 2.75$ & $\mathrm{t}=1.052, \mathrm{p}=0.295$ \\
\hline $\begin{array}{l}\text { Mean gestational age } \\
\text { (in weeks) }\end{array}$ & $38.15 \pm 0.5$ & $38.15 \pm 0.4$ & $\chi^{2}=2.646, \mathrm{p}=0.266$ \\
\hline Primary caesarean & $45(62.5 \%)$ & $37(51.4 \%)$ & $\chi^{2}=1.813, \mathrm{p}=0.178$ \\
\hline Cases with previous caesarean section & $\mathrm{N}=27$ & $\mathrm{~N}=35$ & $\chi^{2}=1.077, \mathrm{p}=0.299$ \\
\hline $\begin{array}{l}\text { Mean duration of ruptured membranes } \\
\text { (in hrs) }\end{array}$ & $1.31 \pm 2.31$ & $0.82 \pm 2.35$ & $\mathrm{t}=1.251, \mathrm{p}=0.213$ \\
\hline $\begin{array}{l}\text { Mean no. of pelvic examinations before } \\
\text { delivery }\end{array}$ & $2.60 \pm 2.45$ & $1.28 \pm 1.60$ & $\mathrm{t}=3.814, \mathrm{p}<0.001$ \\
\hline $\begin{array}{l}\text { Mean pre-operative hemoglobin } \\
\text { (Hb) } \pm \text { SD }\end{array}$ & $11.13 \pm 1.26$ & $11.29 \pm 1.26$ & $\mathrm{t}=0.739, \mathrm{p}=0.461$ \\
\hline Mean post-operative Hb \pm SD $(\mathbf{g} / \mathbf{d l})$ & $9.96 \pm 1.24$ & $10.20 \pm 1.20$ & $\mathrm{t}=1.177, \mathrm{p}=0.241$ \\
\hline $\begin{array}{l}\text { Mean blood loss during caesarean } \\
\text { operation } \pm \text { SD }(\mathbf{m l})\end{array}$ & $793 \pm 101$ & $803 \pm 103$ & $\mathrm{t}=0.613, \mathrm{p}=0.541$ \\
\hline
\end{tabular}

Table 2: Post-operative infectious morbidity in cases with intact membranes at caesarean section between two groups.

\begin{tabular}{|llll|}
\hline Variables & Group A $(\mathbf{N}=49)(\%)$ & Group B $(\mathbf{N}=60)(\%)$ & Statistical significance \\
\hline Fever & $1(2.0)$ & $10(16.7)$ & $\chi^{2}=6.359, p=0.012$ \\
\hline Endometritis & 0 & $7(11.7)$ & $\chi^{2}=6.109, \mathrm{p}=0.013$ \\
\hline Seroma & $1(2.0)$ & $10(16.7)$ & $\chi^{2}=6.359, \mathrm{p}=0.012$ \\
\hline Gaping of stitch line & $2(3.9)$ & $10(16.7)$ & $\chi^{2}=4.914, \mathrm{p}=0.027$ \\
\hline Foul smelling lochia & $1(2.0)$ & $10(16.7)$ & $\chi^{2}=6.359, \mathrm{p}=0.012$ \\
\hline UTI & $1(2.0)$ & $5(8.3)$ & $\chi^{2}=2.053, \mathrm{p}=0.152$ \\
\hline Hospital readmission & $1(2.0)$ & $4(6.7)$ & $\chi^{2}=1.319, \mathrm{p}=0.251$ \\
\hline Re-sutured wound & $4(8.2)$ & $13(21.7)$ & $\chi^{2}=3.736, \mathrm{p}=0.053$ \\
\hline Burst abdomen & 0 & 0 & - \\
\hline
\end{tabular}

Table 3: Comparison of post-operative infectious morbidity in group A and B with ruptured membranes at caesarean section.

\begin{tabular}{|llll|}
\hline Variables & Group A $(\mathbf{N}=\mathbf{2 3})(\%)$ & Group $\mathbf{B}(\mathbf{N}=\mathbf{1 2})(\%)$ & Statistical significance \\
\hline Fever & $3(13.0)$ & $1(8.3)$ & $\chi^{2}=0.173, p=0.678$ \\
\hline Endometritis & $2(8.7)$ & $1(8.3)$ & $\chi^{2}=0.001, p=0.97$ \\
\hline Seroma & $3(13.0)$ & 0 & $\chi^{2}=1.712, p=0.191$ \\
\hline Gaping of stitch line & $3(13.0)$ & $1(8.3)$ & $\chi^{2}=0.173, p=0.678$ \\
\hline Foul smelling lochia & $2(8.7)$ & $1(8.3)$ & $\chi^{2}=0.001, p=0.971$ \\
\hline UTI & $1(4.3)$ & $0(0)$ & $\chi^{2}=0.537, p=0.464$ \\
\hline Re-sutured wound & $4(17.4)$ & $2(16.7)$ & $\chi^{2}=0.003, p=0.957$ \\
\hline
\end{tabular}


Table 4: Comparison of overall post-operative infectious morbidity in group A and B.

\begin{tabular}{|llll|}
\hline Variables & Group A $(\mathbf{N}=\mathbf{7 2})(\%)$ & Group B $(\mathbf{N}=72)(\%)$ & Statistical significance \\
\hline Fever & $4(5.6)$ & $11(15.3)$ & $\chi^{2}=3.647, p=0.056$ \\
\hline Endometritis & $2(2.8)$ & $8(11.1)$ & $\chi^{2}=3.869, \mathrm{p}=0.049$ \\
\hline Seroma & $4(5.6)$ & $10(13.9)$ & $\chi^{2}=2.848, \mathrm{p}=0.091$ \\
\hline Gaping of stitch line & $5(6.9)$ & $11(15.3)$ & $\chi^{2}=2.531, \mathrm{p}=0.112$ \\
\hline Foul smelling lochia & $3(4.2)$ & $11(15.3)$ & $\chi^{2}=5.063, \mathrm{p}=0.024$ \\
\hline UTI & $2(2.8)$ & $5(6.9)$ & $\chi^{2}=1.351, \mathrm{p}=0.245$ \\
\hline Hospital readmission & $1(1.4)$ & $4(5.6)$ & $\chi^{2}=1.865, \mathrm{p}=0.172$ \\
\hline Re-sutured wound & $8(11.1)$ & $15(20.8)$ & $\chi^{2}=2.535, \mathrm{p}=0.111$ \\
\hline Burst abdomen & 0 & 0 & - \\
\hline
\end{tabular}

\section{DISCUSSION}

This was a randomized controlled trial to assess the efficacy of preoperative vaginal wash with $1 \%$ povidone iodine with regard to the caesarean post-operative infectious morbidity. 72 women were in the experimental group $\mathrm{A}$ and 72 women in the control group B.

In our study the overall incidence of endometritis was $2.8 \%$ in the experimental group versus $11.1 \%$ in the control group which was statistically significant, $\mathrm{p}$ value $<0.049$ (Table 4).

Memon et al also found a similar trend with higher incidence of endometritis in the control group, i.e.; 7\%, versus $1 \%$ in the experimental group, statistically significant, with p value $<0.05 .{ }^{8}$ In contrast, Asghania et al found the incidence of endometritis as $0.4 \%$ and $2.5 \%$ in the experimental group and the control group respectively but the difference did not reach statistical significance. ${ }^{9}$

The results of the overall infectious morbidity of this study show that foul smelling discharge was seen in $4.2 \%$ in the experimental group in comparison to $15.3 \%$ in the control group which was statistically significant, $\mathrm{p}$ value $=0.024$ (Table 4).

Fever, seroma, gaped incisional wound, need to re-suture the wound, urinary tract infection, hospital re-admission was less in the experimental group in comparison with the control group, though in these parameters statistical significance was not reached. This study found that preoperative vaginal wash with $1 \%$ povidone iodine was more beneficial in reducing postoperative morbidity in cases where the membranes were intact at the time of caesarean section than with those where the membranes had ruptured at the time of caesarean section.

Febrile morbidity was $2 \%$ in group A, and $16.7 \%$ in group $\mathrm{B}$, in cases with intact membranes, the difference statistically significant with $\mathrm{p}$ value 0.012 (Table 2). Similarly, the difference in the infectious morbidity parameters between the experimental and the control group subjects with intact membranes, with regard to endometritis, seroma, gaped incisional site, foul smelling discharge was statistically significant with $\mathrm{p}$ value 0.013 , 0.012, 0.027, 0.012 (Table 2).

But, interestingly, in our study no significant statistical difference for post-operative infectious morbidity was seen in the Experimental and the control group amongst women who had ruptured membranes at the time of caesarean section.

However, Yildirim et al in their study reported that among the women with ruptured membranes, the incidence of different post-operative morbidity outcomes was lower in the experimental as compared to non-experimental group and found the difference statistically significant for endometritis. This is in contrast to our observations. ${ }^{12}$ Yildirim et al did not mention the concentration of povidone iodine used. The difference in the result could be attributed to differences in the concentration of povidone iodine solution used. ${ }^{12}$ Kaur et al also observed that endometritis rate was significantly lower in the experimental group as compared to control group among the women with ruptured membranes. ${ }^{11}$

Though in our study, statistical significance was reached for some of the parameters of infectious morbidity but, when correlated with other studies substantiate the promising role of povidone iodine vaginal preparation as a preventive measure to reduce post-operative infectious morbidity in women undergoing caesarean section.

\section{CONCLUSION}

Every effort needs to be done to reduce the caesarean postoperative infectious morbidity. The use of preoperative vaginal cleansing with $1 \%$ povidone iodine was one such effort. The findings of this study showed a positive impact of vaginal preparation with $1 \%$ povidone iodine solution prior to caesarean delivery, particularly for endometritis, unhealthy lochia with special reference to women who had intact membranes at the time of caesarean delivery. In this study, statistical significance for each of the post-operative infectious morbidity was not obtained which was also observed in other studies. It can be concluded that there is a limited beneficial role of vaginal preparation with $1 \%$ povidone iodine prior to caesarean 
delivery. Larger sample size randomized controlled studies could help in understanding the impact of this intervention.

Funding: No funding sources

Conflict of interest: None declared

Ethical approval: The study was approved by the Institutional Ethics Committee

\section{REFERENCES}

1. Olsen MA, Butler AM, Willers DM, Devkota P, Gross GA, Fraser VJ. Risk factors for surgical site infection after low transverse cesarean section. Infect Control Hosp Epidemiol. 2008;29(6):477-84.

2. Kofman N, Sheiner E, Levy A, Holcberg G. Risk factors for wound infection following cesarean deliveries. Int J Gynaecol Obstet. 2005;90(1):10-5.

3. Opoien HK, Valbo A, Grinde AA, Walberg M. Postcesarean surgical site infections according to CDC standards: rates and risk factors. A prospective cohort study. Acta Obstet Gynecol Scand. 2007;86(9):1097102.

4. Haas DM, Morgan S, Contreras K, Enders S. Vaginal preparation with antiseptic solution before cesarean section for preventing postoperative infections. Cochrane Database Syst Rev. 2018;7(7):7892.

5. Lumbiganon P, Laopaiboon M, Gulmezoglu AM, Souza JP, Taneepanichskul S, Ruyan P, et al. Method of delivery and pregnancy outcomes in Asia: the WHO global survey on maternal and perinatal health 2007-08. Lancet. 2010;375(9713):490-9.

6. Torres SI, Umscheid CA, Bratzler DW, Leas B, Stone EC, Kelz RR, et al. Centers for Disease Control and
Prevention Guideline for the Prevention of Surgical Site Infection, 2017. JAMA Surg. 2017;152(8):78491.

7. WHO. WHO recommendations for prevention and treatment of maternal peripartum infections, 2015. Available at: https://www.who.int/reproductivehealth/publication maternalperhealth/peripartum-infectionguidelines/en. Accessed on 9 May 2021.

8. Memon S, Qazi RA, Bibi S, Parveen N. Effect of preoperative vaginal cleansing with an antiseptic solution to reduce post caesarean infectious morbidity. J Pak Med Assoc. 2011;61(12):1179-83.

9. Asghania M, Mirblouk F, Shakiba M, Faraji R. Preoperative vaginal preparation with povidoneiodine on post-caesarean infectious morbidity. J Obstet Gynaecol. 2011;31(5):400-3.

10. Aref NK. Vaginal cleansing prior to caesarian section: To do or not to do?: A randomized trial. J Gynecol Obstet Human Reprod. 2019;48(1):6568.

11. Kaur TP, Rathore AM, Sahu L. Efficacy of betadine vaginal toileting before caesarean section in postoperative infections. Int $\mathrm{J}$ Reprod Contracept Obstet Gynecol. 2020;9(6):2457-62.

12. Yildirim G, Gungorduk K, Asicioglu O, Basaran T, Temizkan O, Davas I, et al. Does vaginal preparation with povidone-iodine prior to caesarean delivery reduce the risk of endometritis? A randomized controlled trial. J Matern Fetal Neonatal Med. 2012;25(11):2316-21.

Cite this article as: Chandra S, Singh V. Vaginal preparation with povidone iodine solution before caesarean section and its impact on the post-operative infectious morbidity. Int J Reprod Contracept Obstet Gynecol 2021;10:2775-9. 